\title{
Nonmuscle Myosin II helps regulate synaptic vesicle mobility at the Drosophila neuromuscular junction
}

\author{
Sara Seabrooke ${ }^{\dagger}$, Xinping Qiu $^{\dagger}$, Bryan A Stewart
}

\begin{abstract}
Background: Although the mechanistic details of the vesicle transport process from the cell body to the nerve terminal are well described, the mechanisms underlying vesicle traffic within nerve terminal boutons is relatively unknown. The actin cytoskeleton has been implicated but exactly how actin or actin-binding proteins participate in vesicle movement is not clear.

Results: In the present study we have identified Nonmuscle Myosin II as a candidate molecule important for synaptic vesicle traffic within Drosophila larval neuromuscular boutons. Nonmuscle Myosin II was found to be localized at the Drosophila larval neuromuscular junction; genetics and pharmacology combined with the timelapse imaging technique FRAP were used to reveal a contribution of Nonmuscle Myosin II to synaptic vesicle movement. FRAP analysis showed that vesicle dynamics were highly dependent on the expression level of Nonmuscle Myosin II.

Conclusion: Our results provide evidence that Nonmuscle Myosin II is present presynaptically, is important for synaptic vesicle mobility and suggests a role for Nonmuscle Myosin II in shuttling vesicles at the Drosophila neuromuscular junction. This work begins to reveal the process by which synaptic vesicles traverse within the bouton
\end{abstract}

\section{Background}

Transport and assembly of synaptic vesicles has been the subject of several studies. Vesicles and their components are transported along axon microtubules to the nerve terminal, (for review see $[1,2]$ ) where they participate in synaptic physiology, undergoing a cycle of exoand endocytosis. However, vesicle traffic within terminal boutons is not well understood although recent advances in this area have been made [3,4].

Classically, vesicles were believed to be relatively stationary until released [5-7]. However, more recent studies provided evidence for a mobile vesicle pool $[8,9]$ best described by a caged-diffusion model [10] and differential vesicle mobility in the reserve and recycling pool has been suggested within the frog motor nerve terminals [11]. Additionally, Nunes et al. [12] observed

\footnotetext{
* Correspondence: bryan.stewart@utoronto.ca

† Contributed equally

Department of Biology, University of Toronto, Mississauga, ON L5L 1C6, Canada
}

(c) 2010 Seabrooke et al; licensee BioMed Central Ltd. This is an Open Access article distributed under the terms of the Creative Commons Attribution License (http://creativecommons.org/licenses/by/2.0), which permits unrestricted use, distribution, and reproduction in any medium, provided the original work is properly cited. and dynamic vesicles have been reported at ribbon synapses in lizards [13].

Vesicle movement may result from diffusion or directed transport. Actin polymerization in the nerve terminal may promote vesicle movement through a Listeria comet mechanism [14] or it may act as a substrate for myosin motors to shuttle vesicles. In light of a previous screen from our lab identifying Nonmuscle Myosin II (NMMII) as a candidate molecule important in neuromuscular junction (NMJ) development $[15,16]$, we have focused on determining a neuromuscular function for this actin-based myosin motor. NMMII is present in the nervous system of Xenopus, mouse, rat and chicken [17-19], and in the CNS of Drosophila [20]. NMMII can both crosslink F-actin and has been shown to transport vesicles on F-actin [21-23].

This study was undertaken to determine whether NMMII contributes to synaptic vesicle mobility. We used the genetic model system Drosophila melanogaster 
to manipulate NMMII expression, pharmacology to inhibit NMMII activity, and the optically accessible neuromuscular synapse of third instar larvae to investigate vesicle dynamics. We show that NMMII is concentrated pre- and postsynaptically and importantly, we report that unstimulated synaptic vesicle mobility exhibited a dependence on NMMII expression. These results report the first evidence for NMMII having a function in synaptic vesicle dynamics at the Drosophila NMJ.

\section{Results}

Nonmuscle Myosin II is localized pre- and postsynaptically at the NMJ

Although NMMII has been previously found in the Drosophila CNS [20], it has not been previously localized to the NMJ. Therefore we used immunocytochemistry to investigate the localization of NMMII at the NMJ (Figure. 1). First, we demonstrated the presence of NMMII by staining the NMJ with anti-NMMII and found a robust signal labelling the nerve terminal, which colocalized to a high degree with anti-HRP, a general marker of insect neural membrane. We did notice however that some of the NMMII staining was found outside the boutons defined by the anti-HRP signal suggesting that some of the NMMII signal has a postsynaptic origin. To further investigate this possibility we double labelled the NMJ with the post-synaptic marker, Discs large and anti-NMMII. We found colocalization between Discs large and NMMII, indicating NMMII is found postsynaptically. To determine whether NMMII is also found presynaptically, we expressed UASzipRNAi in the muscle using the muscle specific driver 24BGal4. This dramatically reduces NMMII in the muscle, revealing presynaptic NMMII, which colocalizes with the specific neural marker anti-HRP. Together these results demonstrate the presence of NMMII at the Drosophila NMJ.

\section{Expression level of Nonmuscle Myosin II in zipper alleles}

In order to study NMMII we manipulated its expression level using genetic tools. In Drosophila, NMMII is encoded by the zipper (zip) gene. We used the heterozygous loss-of-function $z i p^{1}$ allele $(\mathrm{Het})$, overexpressed zip with the zip transgene, zip ${ }^{G S 50077}(O / E)$, and expressed a zip knockdown construct, zipRNAi $(K / D)$. Before proceeding with using these reagents in experiments we confirmed the different zip tools were functioning as expected by western blot analysis of protein prepared from larval brain extracts (Figure. 2). NMMII expression in $e l a v^{3 A}$ Gal4 (ct) larvae were used as a control and normalized to 1.00. RNAi Knockdown of NMMII significantly reduced the NMMII level to $28 \%(K / D, 0.28 \pm$ $0.05, \mathrm{n}=5$ ) of the control. The heterozygous loss-offunction allele $z i p^{1} / \mathrm{CyO}$ reduced NMMII expression to
$57 \%$ (Het, $0.57 \pm 0.07, \mathrm{n}=4$ ) of the control while overexpression of NMMII increased protein levels by $95 \%$ compared $(O / E, 1.95 \pm 0.25, \mathrm{n}=4)$ to elav $^{3 A}$ Gal4 (ct) (Figure 2). Statistical analysis revealed significant variation among the groups (ANOVA, p < 0.0001), while post hoc Newman-Keuls comparison between groups showed that each group was different from the other (at least $\mathrm{p}<0.05)$ with the exception that $z i p^{1} / C y O$ was not found to be statistically different from the knockdown sample despite the $50 \%$ difference in average protein levels.

\section{Inhibition of Myosin with ML-9 results in a dose-dependent decrease in vesicle mobility}

NMMII has been previously shown to function in NMJ development $[15,16]$. For this reason, we investigated whether NMMII affected vesicle dynamics at the Drosophila NMJ. To assess the effects of NMMII on vesicle dynamics, synaptic vesicle mobility was measured using FRAP under conditions of altered Myosin activity. To visualize synaptic vesicles in vivo, the recombinant

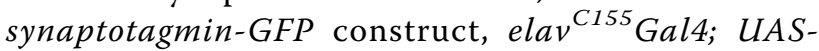
sytGFP, was used to drive expression of synaptotagminGFP in the nervous system. Synaptotagmin-GFP has previously been shown to mark synaptic vesicles $[12,24]$. To begin our examination of myosin activity at the synapse we used ML-9, an inhibitor of myosin light chain kinase (MLCK), and tested synaptic vesicle mobility under different doses of this Myosin inhibitor

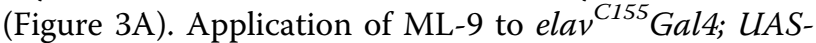
sytGFP/Y larvae resulted in a dose-dependent reduction in the mobility of synaptic vesicles compared to vesicle dynamics in a saline + DMSO control (Figure 3B). Vesicle mobility in this saline + DMSO treated control $(\mathrm{n}=$ 20 boutons, 4 larvae) did not significantly differ from the saline only control ( $\mathrm{n}=22$ boutons from 4 larvae, $\mathrm{P}$ $>0.05$ ) used here in the subsequent FRAP experiments. A significant decrease in vesicle mobility was observed with $100 \mu \mathrm{M}$ ML-9, which resulted in the most dramatic reduction of vesicle mobility $(n=20$ boutons from 4 larvae, $\mathrm{P}<0.05)$ while $50 \mu \mathrm{M}$ ML-9 resulted in slightly, but significantly less vesicle mobility ( $n=20$ boutons from 4 larvae, $\mathrm{P}<0.05)$ compared to the control. A dose of $10 \mu \mathrm{M}$ ML-9 $(\mathrm{n}=20$ boutons from 4 larvae, $\mathrm{P}>0.05$ ) had no effect on vesicle dynamics (Figure $3 \mathrm{~B}$, Table 1 and Additional files 1 and 2 for example movies of vesicle dynamics with control and $100 \mu \mathrm{M}$ ML-9). Together this indicates a dose-dependent response of vesicle mobility to Myosin inhibition. ML-9 is a general Myosin inhibitor while (-)-Blebbistatin is known to be selective towards inhibiting NMMII [25]. However (-)-Blebbistatin is autofluorescent under blue light and is quickly deactivated upon exposure to blue light $[26,27]$ 

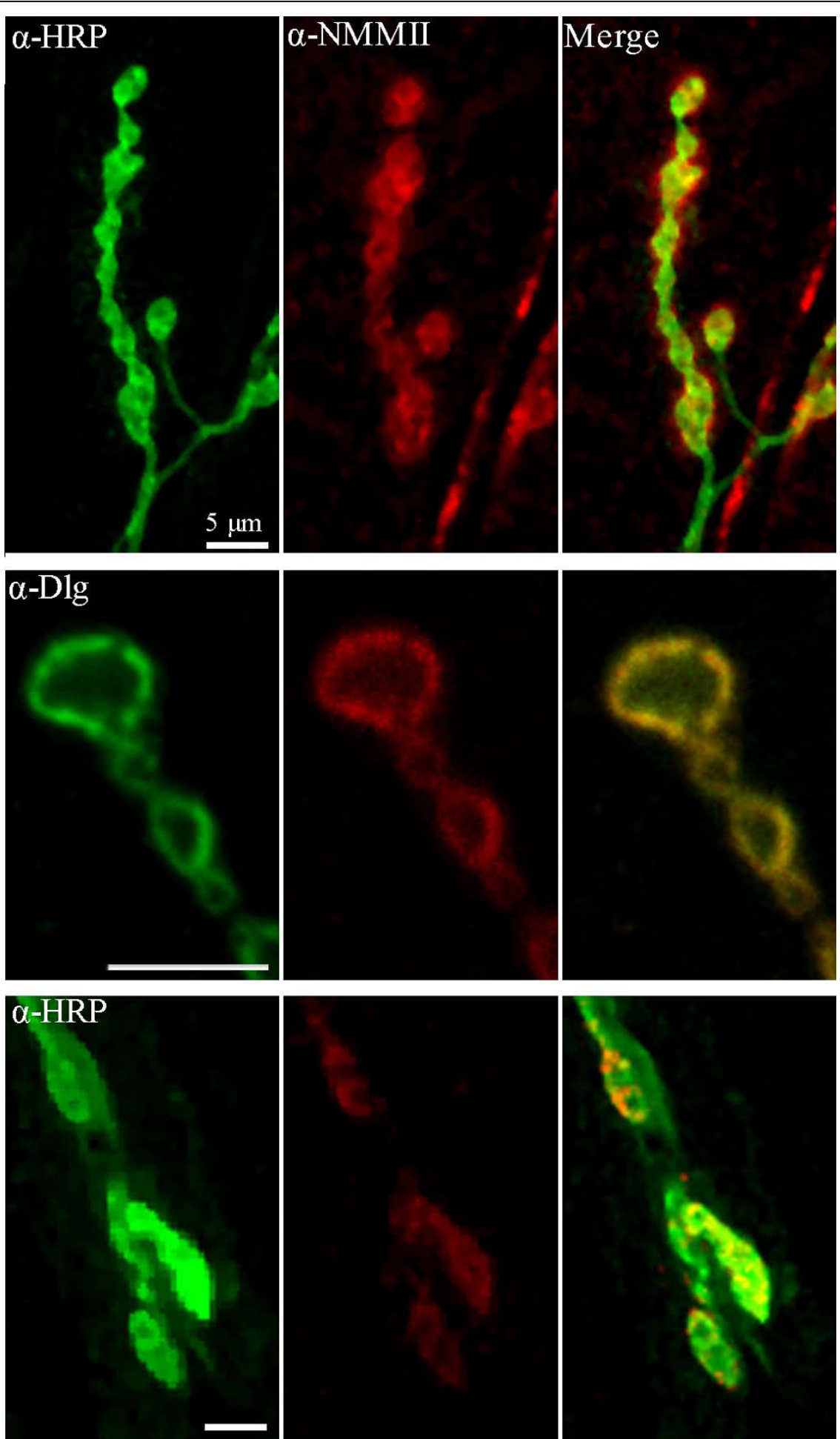

Figure 1 NMMII is localized pre- and postsynaptically at the NMJ. $\alpha$-NMMII (red) was found to colocalize with the neural marker $\alpha$-HRP (green). $\alpha$-NMMII staining is observed which did not colocalize with $\alpha$-HRP (merge) suggesting NMMII is also present postsynaptically (top row). The postsynaptic marker $\alpha$-Dlg (green) is observed to colocalize (merge) with $\alpha$-NMMII (red) suggesting NMMII is present postsynaptically (second row). To confirm presynaptic expression of NMMII, UASzipRNAi was expressed postsynaptically using 24BGal4 (bottom row). This eliminated postsynaptic expression of NMMII. Immunocytochemistry revealed that while postsynaptic staining of NMMII had been eliminated, NMMII was still present presynaptically as seen with the colocalization with $\alpha$-HRP (merge). No postsynaptic NMMIl is visible in the merged image. The top and bottom rows are shown as confocal stacks, while the middle two rows are shown as single confocal slices. 

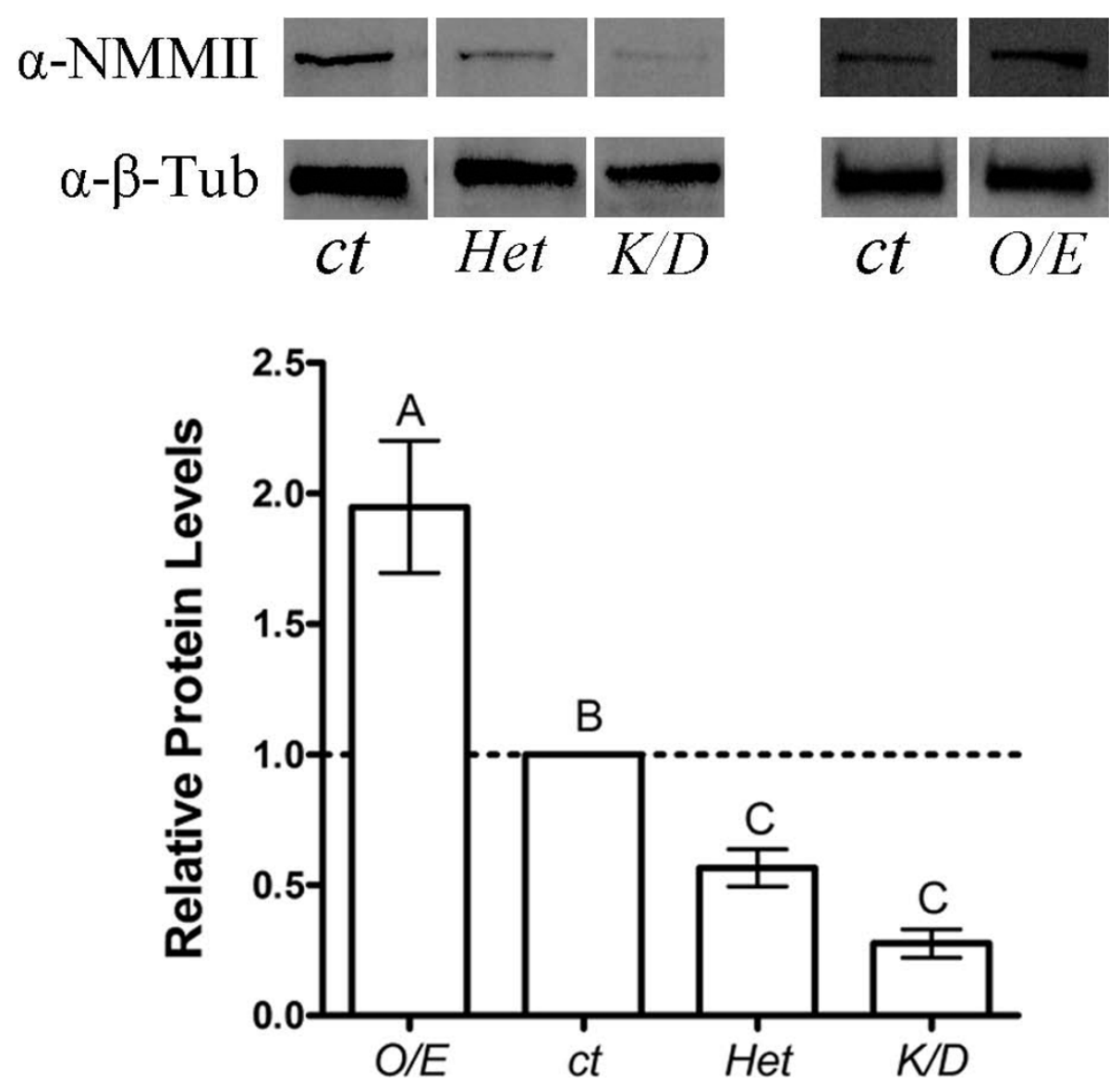

Figure 2 Quantification of NMMII alleles. Western blot of NMMII alleles $a$, Knockdown (KVD) of NMMII in the nervous system reduced NMMII expression to $28 \%(n=5, P<0.05)$ whereas the heterozygous loss-of-function zip $/$ /CyO (Het) reduced NMMIl expression to $57 \%(n=4, P<0.05)$ compared to the elav ${ }^{3 A} \mathrm{Gal} 4$ control $(c t)$. Overexpression of zip ${ }^{G S 50077}(\mathrm{O} / \mathrm{E})$ in the nervous system increased NMMII expression by $95 \%(\mathrm{n}=4, \mathrm{P}<$ 0.05) compared to the elav ${ }^{3 A} G a / 4$ control (ct). $\alpha-\beta$-Tubulin was used as the loading control. Relative NMMII proteins levels were quantified from the western blot analysis. Each band was first normalized to the level of $\beta$-tubulin (used as a loading control, not shown) and then the mutant genotypes were normalized to elav ${ }^{3 A} G$ al4 protein levels, which were set to 1 . The bars indicate the mean level obtained from 4-5 samples, bars are SEM. Overexpression (O/E), knockdown (K/D), heterozygous loss-of-function (Het).

thus limiting our ability to use this drug with our FRAP conditions. We did however obtain NMMII alleles to determine their affect on vesicle mobility.

\section{Nonmuscle Myosin II contributes to synaptic vesicle dynamics}

In order to clarify whether NMMII was contributing to the inhibition of vesicle dynamics by ML-9, we genetically combined elav ${ }^{C 155}$ Gal4; UAS-sytGFP with the NMMII alleles; $z i p^{1}, U A S z i p R N A i$ and $z i p^{G S 50077}$. This allowed us to visualize the affect on vesicle dynamics when NMMII expression was altered (Figure 4A). When NMMII levels are knocked down using elav ${ }^{C 155}$ Gal4; UAS-sytGFP/+; UASzipRNAi/+ $(K / D, \mathrm{n}=26$ boutons from 5 larvae) the rate of vesicle movement is significantly reduced compared to the elav ${ }^{\mathrm{C155}}$ Gal4; UASsytGFP/Y (ct, $\mathrm{n}=19$ boutons from 4 larvae, $\mathrm{P}<0.05)$ control. Surprisingly, vesicle dynamics were also reduced $(O / E, \mathrm{n}=23$ boutons from 5 larvae, $\mathrm{P}<0.0001)$ when

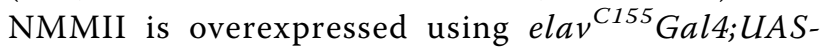

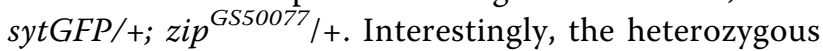
loss-of-function NMMII allele, $z i p^{1} /+$ resulted in enhanced mobility of vesicles (Het, $\mathrm{n}=21$ boutons from 4 larvae, $\mathrm{P}<0.05$ ) (Figure. 4B, Table 1 and Additional files $1,3,4$ and 5 for example movies of vesicle dynamics with NMMII manipulation).

Although the bleach depth was not significantly different between the heterozygous loss-of-function allele, the RNAi knockdown sample and control samples, the $\mathrm{O} / \mathrm{E}$ samples did show significantly lower bleach depth (See Additional file 6, Figure S1A showing the average bleach depth from FRAP experiments). We therefore further analysed the FRAP recoveries to ensure differences in bleach depth did not account for our results. To refine 

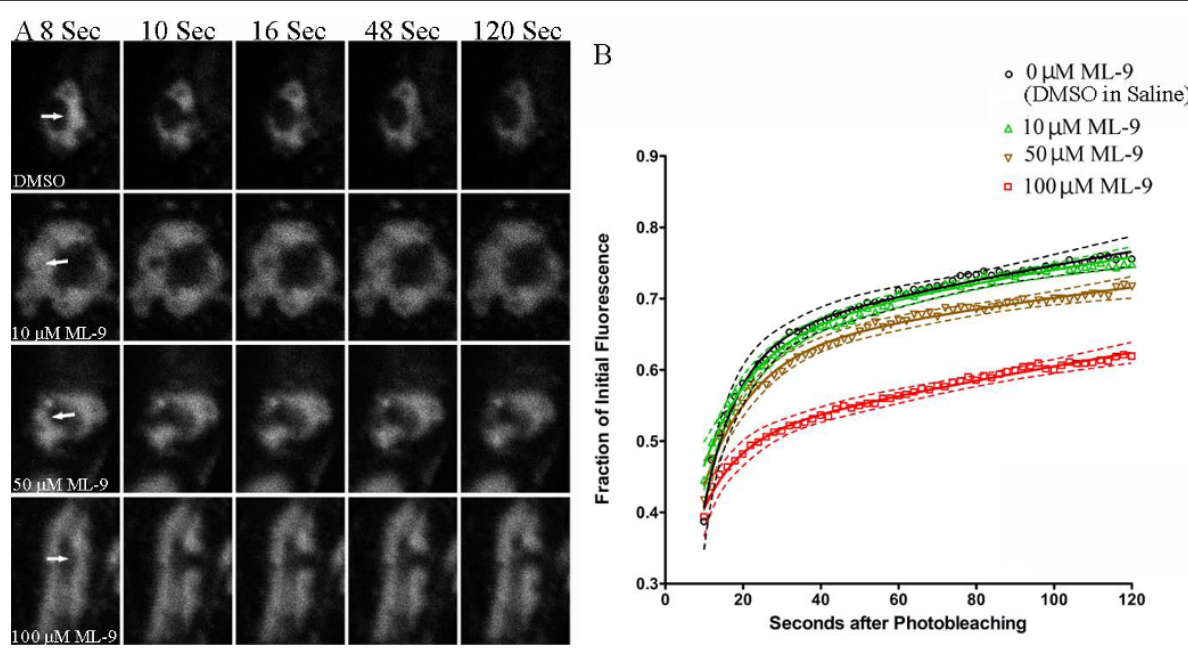

Figure 3 FRAP indicating the mobility of vesicles in response to application of the Myosin inhibitor, ML-9. a, Acquired images for the recovery of fluorescently labelled vesicles using elav ${ }^{C 155}$ Gal4;UAS-sytGFP/Y after application of ML-9. Images are shown immediately before bleaching $(8 \mathrm{sec})$, immediately after photobleaching $(10 \mathrm{sec})$ and at 16, 48 and $120 \mathrm{sec}$. Bleached areas are indicated by white arrows. A reduction in recovery is observed with increasing doses of ML-9. b, A significant dose-dependent response was observed for inhibition with ML9 between the doses of $100 \mu \mathrm{M}$ ML-9 and $10 \mu \mathrm{M}$ ML-9 as compared to $0 \mu \mathrm{M}$ ML-9. $10 \mu \mathrm{M} \mathrm{ML}-9$ did not significantly reduced vesicle mobility. Error is represented as the $95 \%$ confident interval of the curve. FRAP recoveries were fit with double exponential curves and nonlinear regression was used to test for statistical differences. Sample sizes and significance were as follows: $0 \mu M M L-9(n=20), 10 u M ~ M L-9(n=22, p>0.05)$, $50 \mu \mathrm{M}$ ML-9 $(n=20, p<0.05), 100 \mu \mathrm{M} M L-9(n=20, p<0.05)$.

Table 1 Rate parameters from FRAP curves fit with the double exponential curves

\begin{tabular}{|c|c|c|c|c|}
\hline \multirow[t]{2}{*}{ Treatment } & \multicolumn{4}{|c|}{ Rate Parameters $^{1}$} \\
\hline & A & K1 & B & $\mathrm{K} 2$ \\
\hline \multicolumn{5}{|l|}{ Vesicle Mobility } \\
\hline $0 \mu \mathrm{M} M L-9$ & $0.38 \pm 0.031$ & $0.0040 \pm 0.0010$ & $0.72 \pm 0.031$ & $0.11 \pm 0.40$ \\
\hline $10 \mu \mathrm{M} \mathrm{ML}-9$ & $0.37 \pm 0.0035$ & $0.0035 \pm 0.0010$ & $0.40 \pm 0.083$ & $0.080 \pm 0.023$ \\
\hline $50 \mu \mathrm{M} M L-9$ & $0.39 \pm 0.030$ & $0.0025 \pm 0.0010$ & $0.42 \pm 0.087$ & $0.080 \pm 0.022$ \\
\hline $100 \mu \mathrm{M}$ ML-9 & $0.51 \pm 0.020$ & $0.0025 \pm 0.0005$ & $0.29 \pm 0.16$ & $0.11 \pm 0.050$ \\
\hline Control (ct) & $0.40 \pm 0.033$ & $0.0047 \pm 0.0012$ & $0.58 \pm 0.22$ & $0.10 \pm 0.039$ \\
\hline Heterozygous Loss-of-Function (Het) & $0.37 \pm 0.043$ & $0.0069 \pm 0.0015$ & $0.66 \pm 0.33$ & $0.12 \pm 0.049$ \\
\hline RNAi Knockdown (KV) & $0.46 \pm 0.035$ & $0.0031 \pm 0.0008$ & $0.28 \pm 0.12$ & $0.082 \pm 0.045$ \\
\hline Overexpression (O/E) & $0.49 \pm 0.040$ & $0.0023 \pm 0.0010$ & $0.35 \pm 0.093$ & $0.071 \pm 0.030$ \\
\hline
\end{tabular}

${ }^{1}$ Equation: $\operatorname{Frap}(\mathrm{t})=1-\mathrm{A}\left(\mathrm{e}^{-\mathrm{K} 1}\right.$ off $\left.\mathrm{t}\right)-\mathrm{B}\left(\mathrm{e}^{-\mathrm{K} 2 \text { off } \mathrm{t}}\right)[38]$ where $\mathrm{A}, \mathrm{B}=$ constants that represent the apparent bleached fraction, $\mathrm{K} 1, \mathrm{~K} 2=$ rates, $\mathrm{t}=$ time

our analysis, we calculated the recovery index of the FRAP curve [12] which accounts for differences in bleach depth between genotypes. These calculations led to the same conclusions: the heterozygous loss-of-function allele of NMMII increased the recovery index, while both the RNAi knockdown and overexpression of NMMII reduced the recovery index (See Additional file 6, Figure S1B showing the recovery index from the FRAP curves).

Overall these results indicate that NMMII contributes to vesicle mobility in a manner which is highly dependent on the expression level of NMMII (Table 2).

\section{Discussion}

This report has identified the presence of NMMII in the presynaptic terminal and indicates a function for NMMII in synaptic vesicle mobility at the NMJ of Drosophila melanogaster. NMMII has been implicated in synaptic transmission in rats [19], but has not previously been shown at the NMJ of Drosophila and this is the first evidence of NMMII having a function in synaptic vesicle mobility. Using Drosophila, as a genetically malleable tool, and the confocal imaging technique, FRAP, we were able to quantify the effect of NMMII on vesicle mobility. FRAP revealed that NMMII plays a complex 


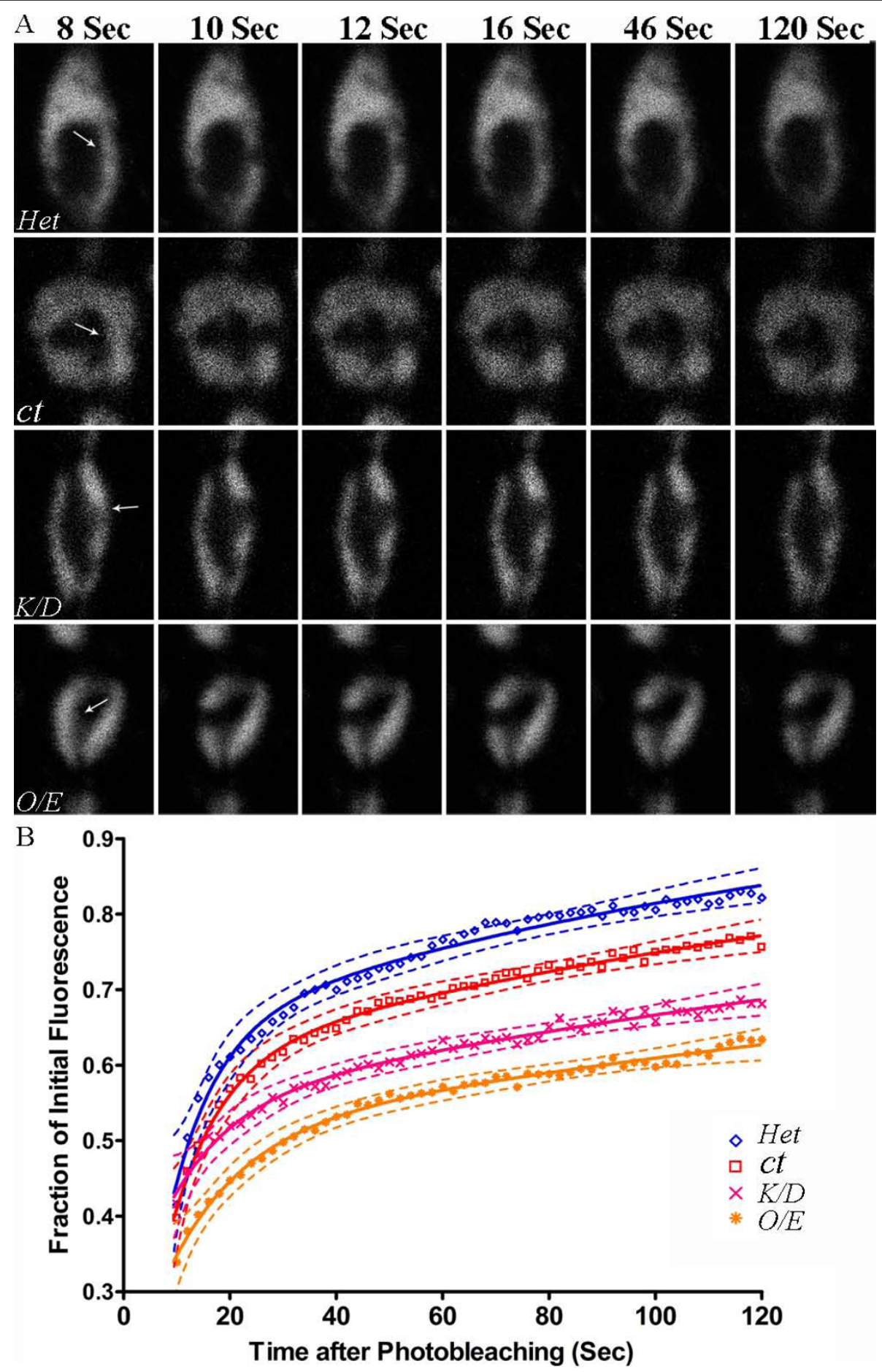

Figure 4 FRAP recovery curves for vesicle mobility when NMMII expression is altered. $a$, Acquired images for the recovery of the heterozygous loss-of-function, elav ${ }^{155} \mathrm{Gal4}$; UAS-sytGFP/+; zip $1+$ + (Het), the control, elav ${ }^{C 155} \mathrm{Gal4}$;UAS-sytGFP/Y (ct), the RNAi knockdown,

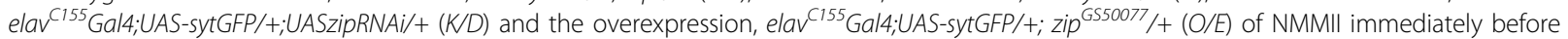
bleaching (8 sec), immediately after photobleaching (10 sec) and at 12,16, 48 and $120 \mathrm{sec}$ post-bleaching. Bleached areas are indicated with white arrows. At $16 \mathrm{sec}$ the bleached region for Het is no longer visible; however, it is still clearly visible in control (ct). At $120 \mathrm{sec}$, the bleached region is no longer visible in the control (ct), but is still visible for both KVD and O/E. b, Vesicle mobility is affected by the expression level of NMMII. FRAP curves reveal that the heterozygous NMMII loss-of-function (Het) significantly enhanced vesicle mobility as compared to the control $(c t)$ while both knockdown (KDD) and overexpression (O/E) of NMMII significantly reduced vesicle mobility. FRAP recoveries were fit with double exponential curves. Nonlinear regression was used to test for statistical differences; elav ${ }^{C 155}$ Gal4;UAS-sytGFPN ( $\left.n=26\right)$, elav ${ }^{C 155}$ Gal4; UAS-sytGFP/+;

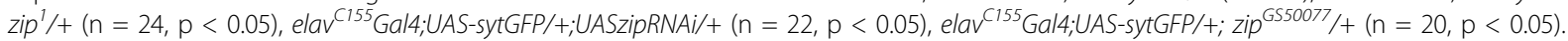


Table 2 Summary of results from manipulating NMMII expression

\begin{tabular}{lcc}
\hline NMMII Allele & Expression of NMMII & Vesicle Mobility \\
\hline \multicolumn{2}{c}{$\uparrow$} & $\downarrow$ \\
\hline O/E & $\downarrow$ \\
\hline Het & $\uparrow$ \\
\hline \multicolumn{2}{c}{} \\
$\begin{array}{l}\uparrow=\text { increased relative to control, } \downarrow=\text { decreased relative to control, } \\
\downarrow \downarrow=\text { further decreased relative to control }\end{array}$
\end{tabular}

role in vesicle dynamics and begins to clarify our knowledge of how synaptic vesicles may be available for release upon stimulation.

\section{Vesicle mobility is affected by a complex interaction with} Nonmuscle Myosin II

Immunocytological staining first identified that NMMII is found both pre- and postsynaptically in the Drosophila NMJ. Abolishment of postsynaptic NMMII expression through RNAi confirmed expression of NMMII presynaptically. With NMMII present in the presynaptic terminal, this suggests a possible function for NMMII in trafficking vesicles within the bouton. To determine whether NMMII impacts vesicle dynamics, we carried out in vivo imaging techniques to visualize synaptic vesicle mobility. We found an intriguing complex interaction between the expression level of NMMII and the dynamics of vesicle mobility. Inhibiting MLCK reduced vesicle mobility, consistent with Jordan et al. [10]. More specifically, increasing either NMMII expression by $95 \%$ or reducing NMMII expression to $28 \%$ reduced vesicle mobility, while moderately reducing NMMII to $57 \%$ enhanced vesicle mobility. A limitation of the present study is an estimate of NMMII activity or levels specifically at nerve terminal boutons in the various mutant strains used. The postsynaptic presence of NMMII makes immunohistochemical techniques for measuring presynaptic NMMII difficult. We estimated neuronal NMMII levels using western blots of larval brains. This demonstrated that NMMII levels were up- and downregulated significantly by the genotypes used. The $50 \%$ reduction in protein level from the heterozygous loss-offunction to the RNAi knockdown strains did not exhibit a statistical difference. However, we did find substantially different effects on vesicle mobility suggesting that the apparent difference in expression levels is functionally significant. Thus it appears that tight regulation of NMMII expression is essential in maintaining appropriate vesicle dynamics: a small reduction in NMMII levels enhances mobility whereas too much or too little impairs mobility. Together this supports a role for NMMII in normal synaptic vesicle mobility at the Drosophila NMJ.

While these findings suggest a role for NMMII in synaptic vesicle mobility, it does not exclude the possibility that other myosin motors are also involved. In support of this, the general myosin inhibitor, ML-9, reduced synaptic vesicle mobility in a dose dependent manner with no stimulation of vesicle mobility at low concentrations. In addition, other myosin motors have been shown to associate with synaptic vesicles and be involved in synaptic transmission. For example, Myosin $\mathrm{V}$ can bind to a myosin receptor found on a subpopulation of high density vesicles [28] and is found to be associated with vesicles isolated from chick brain [29], but was not found to alter hippocampal synaptic transmission in mice [30]. However, Myosin II has been associated with normal synaptic transmission. In cultured rat superior cervical ganglion neurones, myosin IIb was found to inhibit synaptic transmission [19]. A reduction in synaptic transmission upon inhibition of Myosin II was also observed in rat cholinergic synapses [31], while MLCK was found to be involved in maintaining repetitive synaptic transmission [32]. Myosin II has also been shown to be involved in vesicle mobility in other systems. Nonmuscle Myosin II has been shown to transport vesicles on actin filaments in clam oocytes [22] and Nonmuscle Myosin II has been shown to contribute to vesicle transport from the Golgi to the Endoplasmic Reticulum [23]. Thus, while the present work identifies a function for NMMII in synaptic vesicle mobility, the precise mechanisms of myosin motors in synaptic transmission and the precise role of NMMII as a vesicle motor remains to be clarified. Since NMMII interacts with actin, it will also be important to investigate the affects of NMMII on actin stability and dynamics in the NMJ and to determine whether the affects of NMMII on vesicle mobility translate into affects on synaptic transmission.

\section{Conclusions}

Our results show that NMMII is found presynaptically at the Drosophila NMJ and plays a functional role at the NMJ. We report, for the first time, a function for NMMII in normal synaptic vesicle mobility in the unstimulated neuron, which is dependent on the expression level of NMMII. Further experimentation to address the function of NMMII at the NMJ, through electrophysiological assays, manipulating NMMII activity by its' kinases and phosphatases and measuring actin activity, is required to more clearly define the precise role of NMMII at the Drosophila NMJ. 


\section{Methods \\ Drosophila Stocks}

Drosophila melanogaster were maintained at $22^{\circ} \mathrm{C}$ on Bloomington fly media. The heterozygous loss-of-function NMMII allele, zipper ${ }^{1} / C y O \quad\left(z^{1} p^{1} / C y O\right)$ (FBal0018862) was acquired from the Bloomington stock center and rebalanced over Cyo-GFP. UASzipperRNAi (UASzipRNAi) was obtained from the Vienna Drosophila RNAi center (FBst0470845) and is a NMMII RNAi construct. The gain-of-function NMMII construct, zipper $^{G S 50077}\left(\right.$ zip $\left.^{G S 50077}\right)$, was obtained from the Drosophila gene search project [33] and is a unidirectional UAS construct inserted upstream of NMMII. elav ${ }^{\text {C155- }}$

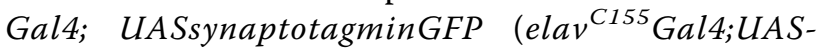
sytGFP) was obtained from the Bloomington stock center (FBst0006923) and UASactinGFP (UAS-actGFP) was obtained from the Kyoto stock center for FRAP imaging of vesicle and actin dynamics respectively. A stock of UAS-actGFP/UAS-actGFP; lav $^{3 A}$ Gal4/TM3, Sb, Tb was generated to express actinGFP in the nervous system. The Gal4 drivers, elav ${ }^{3 A}$ Gal4 (FBti0072910, Bloomington stock center) and elav ${ }^{\mathrm{C} 155}$ Gal4 drive expression of UAS constructs in the nervous system [34]. 24BGal4 (FBti0002090, Bloomington stock center) was used to drive expression of NMMII alleles in muscle. Flies were crossed at $25^{\circ} \mathrm{C}$ and kept at identical growing conditions.

\section{Immunocytochemistry}

Third instar larva were dissected in HL3 buffer with no $\mathrm{Ca}^{2+}$ added [35], and fixed in 4\% paraformaldehyde in phosphate-buffered saline (PBS). After fixation, the samples were transferred to $1 \%$ BSA diluted in PBT (PBS plus $0.1 \%$ Triton $\mathrm{X}-100)$. The following primary antibodies were used: rabbit anti-NMMII (1:1000, Roger Karess, Centre de National de Récherche Scientifique, Centre de Génétique Moléculaire), and mouse anti-Dlg (1:200, Developmental Studies Hybridoma Bank). AntiHRP FITC (1:1000, MP Biomedical; Solon, OH) was used as a neural marker. The secondary antibodies used were; goat anti-mouse Alexa488 and goat anti-rabbit Alexa633 (1:1000, Invitrogen). Following antibody labelling, the preparations were washed in PBT and mounted in Vectashield (Vector Laboratories; Burlington, ON).

Images were acquired on a Carl Zeiss LSM510 confocal. Z-sections were obtained on a F-Fluar $40 \times / 1$.3 Oil for NMMII localization. The pinhole aperture acquired images of $1 \mu \mathrm{m}$ thickness and image sections were projected onto a single plane. 1-3 images were collected per larva.

\section{Western blot}

For each treatment, five brains from third instar larvae were lysed in protein lysis buffer $(50 \mathrm{mM}$ Tris- $\mathrm{Cl}, 1 \%$
$\mathrm{NP}-40,150 \mathrm{mM} \mathrm{NaCl}$ ) with complete protease inhibitor. Samples were separated on a 10\% SDS-polyacrylamide gel, and transferred to PVDF membrane. NMMII was detected with rabbit anti-NMMII antibody (1:10000, Roger Karess, Centre de National de Récherche Scientifique, Centre de Génétique Moléculaire), and a goat anti-rabbit HRP-coupled secondary (1:1500 BioRad). $\alpha$ $\beta$-Tubulin was used as a loading control (1:100, Developmental Studies Hybridoma Bank). Antibodies were visualized using chemiluminescent detection (ECL Plus, Amersham). Control and experimental bands were imaged either simultaneous or individually on the same blot. Blots were scanned and digitized with a Molecular Dynamics Phosphoimager. Band intensities were quantified using ImageJ.

\section{Fluorescence Recovery After Photobleaching}

FRAP was conducted on a Carl Zeiss LSM 510 confocal microscope equipped with an Argon2 laser and a LP505 filter. To immobilize the preparation, wandering third instar larvae were dissected in HL3 with no $\mathrm{Ca}^{2+}$ added [35] and glued to slygard-coated slides using Nexabrand tissue glue (WPI, Sarasota, FL). The glued preparations were placed under an Achroplan 100×/1.0 W Ph objective with an $8 \times$ digital zoom. FRAP recordings were made, from segments 3 or 4 , of type I boutons at muscle $7 / 6$ for vesicle dynamics. Images were collected at $1.12 \mu \mathrm{s} /$ pixel with a pinhole of 1 airy unit and a resolution of $512 \times 512$. Sixty images were collected over two minutes with a $1 \mathrm{sec}$ delay between image acquisitions. To select the area for bleaching, a region of interest (ROI) $24 \times 30$ pixels was selected on the digital image. Four baseline scans were acquired using $5 \%$ or $10 \%$ of full laser power. Before the fifth scan, the laser increased to $97 \%$ of maximal and rapidly iterated the ROI 9 times, after which, returning to $5 \%$ or $10 \%$ of maximal power to complete the remaining 56 scans. A maximum of three type I boutons were recorded per hemi-segment and a maximum of 6 boutons per larvae. All FRAP experiments were completed within 2 hours of the larval dissection.

The acquired image sequences were imported into ImageJ. Images that drifted were digitally stabilized before analysis [36]. The time series analyzer plug-in for ImageJ was used to measure fluorescent intensity. Intensity measurements were taken from the background, the total fluorescence in the bouton, and the fluorescence in the bleached area. A double normalization was completed, as described in [37] using the following equation:

$$
\begin{aligned}
& \left(T_{\text {prebleach }}-B G\right)\left(I_{t}-B G\right) \\
& \left(T_{t}-B G\right)\left(I_{\text {prebleach }}-B G\right)
\end{aligned}
$$


Where $\mathrm{T}=$ total fluorescence in the bouton, $\mathrm{I}=$ fluorescence in the bleached fraction and BG = background fluorescence outside the bouton. This accounts for photobleaching throughout the FRAP experiment and for movement of bleached molecules out of the FRAP ROI.

The recovery curves were fit two a double exponential curve [38] as follows:

$$
\operatorname{Frap}(\mathrm{t})=1-\mathrm{A}\left(\mathrm{e}^{-\mathrm{K} 1 \text { off t }}\right)-\mathrm{B}\left(\mathrm{e}^{-\mathrm{K} 2 \text { off } \mathrm{t}}\right)
$$

Where $\mathrm{A}, \mathrm{B}=$ constants that represent the apparent bleached fraction, $\mathrm{K} 1, \mathrm{~K} 2=$ rates, $\mathrm{t}=$ time.

\section{Drug administration}

To access vesicle dynamics under myosin inhibition, the myosin light chain kinase inhibitor, ML-9, was applied to the preparation before beginning FRAP experiments. A stock concentration of $50 \mathrm{mM}$ ML-9 in DMSO was diluted into HL3 [35] with no $\mathrm{Ca}^{2+}$ added, to make $100 \mu \mathrm{M}, 50 \mu \mathrm{M}$ and $10 \mu \mathrm{M}$ ML-9 solutions. The preparations were incubated in the dark with ML-9 for 30 minutes prior to FRAP.

\section{Statistical analysis}

All statistical analyses were performed in GraphPad Prism 4.0. For non-linear regression, FRAP was double normalized [37] and compiled for curve fitting [38]. In all cases, the double exponential curve outlined in McNally (2008) [38] was accepted over the single exponential curve. Error is represented as the 95\% confidence interval for the curve. Rates are expressed as inverse seconds. In double exponential curves, the initial phase has been indicated here with $\mathrm{A}$ and $\mathrm{K} 1$ and the second phase of the curve have been indicated by $\mathrm{B}$ and K2. For analysis of variance, one-way ANOVA was completed. $\mathrm{P}<0.05$ was accepted as statistically significant.

Additional file 1: Movie 1. FRAP of synaptic vesicles labelled with synaptotagmin-GFP. Time-lapse video recorded over two minutes and compressed into 9 seconds.

Additional file 2: Movie 2. FRAP of labelled synaptic vesicles whose mobility has been inhibited with application of $100 \mu \mathrm{M}$ ML-9. Time-lapse video recorded over two minutes and compressed into 9 seconds.

Additional file 3: Movie 3. FRAP of labelled synaptic vesicles showing that mobility is reduced with RNAi knockdown of NMMII. Time-lapse video recorded over two minutes and compressed into 9 seconds.

Additional file 4: Movie 4. FRAP of labelled synaptic vesicles whose mobility is enhanced with a moderate reduction of NMMII. Time-lapse video recorded over two minutes and compressed into 9 seconds.

Additional file 5: Movie 5. FRAP of synaptotagmin-GFP labelled vesicles showing reduced vesicle mobility with overexpression of NMMII. Timelapse video recorded over two minutes and compressed into 9 seconds.

Additional file 6: Figure S1. Graphs indicating the average bleach depth and the recovery index for the NMMII FRAP experiments.

\section{Acknowledgements}

We acknowledge Owen Randlett for his preliminary work with ML-9, Nicola Haines, Ronald Gonzalez, Colin DeMill and Marta Kisiel for feedback on the manuscript and Nicole Novroski for making fly food. We would like to thank Roger Karess for providing the Nonmuscle Myosin II antibody, the Developmental Studies Hybridoma Bank for antibodies, and the Bloomington, Kyoto, Vienna and Gene Search Stock Centers for fly stocks. This work was supported by funds from the Canadian Institutes of Health Research program to BAS.

\section{Authors' contributions}

SS performed and analysed all FRAP experiments, contributed to conceiving and designing the studies and prepared the manuscript. XQ carried out and analysed western blot data, completed the immunocytochemistry and contributed to the design of the studies. BAS conceived and assisted in designing and conducting the studies, provided feedback and editing of the manuscript and supplied materials and reagents which made these studies possible. All authors read and approved the final manuscript.

Received: 24 September 2009 Accepted: 16 March 2010 Published: 16 March 2010

\section{References}

1. Bennett MK, Scheller RH: A molecular description of synaptic vesicle membrane trafficking. Annual review of biochemistry 1994, 63:63-100.

2. Santos MS, Li H, Voglmaier SM: Synaptic vesicle protein trafficking at the glutamate synapse. Neuroscience 2009, 158(1):189-203.

3. Akbergenova $Y$, Bykhovskaia M: Stimulation-induced formation of the reserve pool of vesicles in Drosophila motor boutons. Journal of neurophysiology 2009, 101(5):2423-2433.

4. Westphal V, Rizzoli SO, Lauterbach MA, Kamin D, Jahn R, Hell SW: Videorate far-field optical nanoscopy dissects synaptic vesicle movement. Science (New York, NY) 2008, 320(5873):246-249.

5. Henkel AW, Simpson LL, Ridge RM, Betz WJ: Synaptic vesicle movements monitored by fluorescence recovery after photobleaching in nerve terminals stained with FM1-43. J Neurosci 1996, 16(12):3960-3967.

6. Kraszewski K, Daniell L, Mundigl O, De Camilli P: Mobility of synaptic vesicles in nerve endings monitored by recovery from photobleaching of synaptic vesicle-associated fluorescence. J Neurosci 1996, 16(19):5905-5913.

7. Miyamoto S: Changes in mobility of synaptic vesicles with assembly and disassembly of actin network. Biochimica et biophysica acta 1995, 1244(1):85-91.

8. Lemke EA, Klingauf J: Single synaptic vesicle tracking in individual hippocampal boutons at rest and during synaptic activity. J Neurosci 2005, 25(47):11034-11044.

9. Shtrahman M, Yeung C, Nauen DW, Bi GQ, Wu XL: Probing vesicle dynamics in single hippocampal synapses. Biophysical journal 2005, 89(5):3615-3627.

10. Jordan R, Lemke EA, Klingauf J: Visualization of synaptic vesicle movement in intact synaptic boutons using fluorescence fluctuation spectroscopy. Biophysical journal 2005, 89(3):2091-2102.

11. Gaffield MA, Rizzoli SO, Betz WJ: Mobility of synaptic vesicles in different pools in resting and stimulated frog motor nerve terminals. Neuron 2006, 51(3):317-325.

12. Nunes $P$, Haines $N$, Kuppuswamy V, Fleet DJ, Stewart BA: Synaptic vesicle mobility and presynaptic $\mathrm{F}$-actin are disrupted in a $\mathrm{N}$-ethylmaleimidesensitive factor allele of Drosophila. Mol Biol Cell 2006, 17(11):4709-4719.

13. Rea R, Li J, Dharia A, Levitan ES, Sterling P, Kramer RH: Streamlined synaptic vesicle cycle in cone photoreceptor terminals. Neuron 2004, 41(5):755-766.

14. Lambrechts A, Gevaert K, Cossart P, Vandekerckhove J, Van Troys M: Listeria comet tails: the actin-based motility machinery at work. Trends in cell biology 2008, 18(5):220-227.

15. Laviolette MJ, Nunes P, Peyre JB, Aigaki T, Stewart BA: A genetic screen for suppressors of Drosophila NSF2 neuromuscular junction overgrowth. Genetics 2005, 170(2):779-792.

16. Peyre JB, Seabrooke S, Randlett O, Kisiel M, Aigaki T, Stewart BA: Interaction of cytoskeleton genes with NSF2-induced neuromuscular junction overgrowth. Genesis 2006, 44(12):595-600. 
17. Kollins KM, Hu J, Bridgman PC, Huang YQ, Gallo G: Myosin-II negatively regulates minor process extension and the temporal development of neuronal polarity. Developmental neurobiology 2009, 69(5):279-298.

18. Ruchhoeft ML, Harris WA: Myosin functions in Xenopus retinal ganglion cell growth cone motility in vivo. Journal of neurobiology 1997, 32(6):567-578.

19. Takagishi Y, Futaki S, Itoh K, Espreafico EM, Murakami N, Murata Y Mochida S: Localization of myosin II and V isoforms in cultured rat sympathetic neurones and their potential involvement in presynaptic function. The Journal of physiology 2005, 569(Pt 1):195-208.

20. Kim YS, Fritz JL, Seneviratne AK, VanBerkum MF: Constitutively active myosin light chain kinase alters axon guidance decisions in Drosophila embryos. Developmental biology 2002, 249(2):367-381.

21. DePina AS, Langford GM: Vesicle transport: the role of actin filaments and myosin motors. Microscopy research and technique 1999, 47(2):93-106.

22. DePina AS, Wollert T, Langford GM: Membrane associated nonmuscle myosin II functions as a motor for actin-based vesicle transport in clam oocyte extracts. Cell motility and the cytoskeleton 2007, 64(10):739-755.

23. Duran JM, Valderrama F, Castel S, Magdalena J, Tomas M, Hosoya H, RenauPiqueras J, Malhotra V, Egea G: Myosin motors and not actin comets are mediators of the actin-based Golgi-to-endoplasmic reticulum protein transport. Molecular biology of the cell 2003, 14(2):445-459.

24. Zhang YQ, Rodesch CK, Broadie K: Living synaptic vesicle marker: synaptotagmin-GFP. Genesis 2002, 34(1-2):142-145.

25. Kovacs M, Toth J, Hetenyi C, Malnasi-Csizmadia A, Sellers JR: Mechanism of blebbistatin inhibition of myosin II. The Journal of biological chemistry 2004, 279(34):35557-35563.

26. Kolega J: Phototoxicity and photoinactivation of blebbistatin in UV and visible light. Biochemical and biophysical research communications 2004, 320(3):1020-1025.

27. Sakamoto T, Limouze J, Combs CA, Straight AF, Sellers JR: Blebbistatin, a myosin II inhibitor, is photoinactivated by blue light. Biochemistry 2005, 44(2):584-588.

28. Miller KE, Sheetz MP: Characterization of myosin V binding to brain vesicles. The Journal of biological chemistry 2000, 275(4):2598-2606.

29. Evans LL, Lee AJ, Bridgman PC, Mooseker MS: Vesicle-associated brain myosin-V can be activated to catalyze actin-based transport. Journal of cell science 1998, 111(Pt 14):2055-2066.

30. Schnell E, Nicoll RA: Hippocampal synaptic transmission and plasticity are preserved in myosin Va mutant mice. Journal of neurophysiology 2001, 85(4):1498-1501.

31. Mochida S, Kobayashi H, Matsuda Y, Yuda Y, Muramoto K, Nonomura Y: Myosin II is involved in transmitter release at synapses formed between rat sympathetic neurons in culture. Neuron 1994, 13(5):1131-1142.

32. Polo-Parada L, Plattner F, Bose C, Landmesser LT: NCAM 180 acting via a conserved C-terminal domain and MLCK is essential for effective transmission with repetitive stimulation. Neuron 2005, 46(6):917-931.

33. Toba G, Ohsako T, Miyata N, Ohtsuka T, Seong KH, Aigaki T: The gene search system. A method for efficient detection and rapid molecular identification of genes in Drosophila melanogaster. Genetics 1999, 151(2):725-737.

34. Brand $\mathrm{AH}$, Perrimon N: Targeted gene expression as a means of altering cell fates and generating dominant phenotypes. Development 1993, 118(2):401-415.

35. Stewart BA, Atwood HL, Renger JJ, Wang J, Wu CF: Improved stability of Drosophila larval neuromuscular preparations in haemolymph-like physiological solutions. J Comp Physiol [A] 1994, 175(2):179-191.

36. Fleet DJ, Weiss Y: Optical flow estimation. Mathematical Models for Computer Vision: The Handbook New York SpringerFaugeras O 2005, 239-258.

37. Phair RD, Gorski SA, Misteli T: Measurement of dynamic protein binding to chromatin in vivo, using photobleaching microscopy. Methods in enzymology 2004, 375:393-414.

38. McNally JG: Quantitative FRAP in analysis of molecular binding dynamics in vivo. Methods in cell biology 2008, 85:329-351.

doi:10.1186/1471-2202-11-37

Cite this article as: Seabrooke et al: Nonmuscle Myosin II helps regulate synaptic vesicle mobility at the Drosophila neuromuscular junction. BMC Neuroscience 2010 11:37.

\section{Submit your next manuscript to BioMed Central and take full advantage of:}

- Convenient online submission

- Thorough peer review

- No space constraints or color figure charges

- Immediate publication on acceptance

- Inclusion in PubMed, CAS, Scopus and Google Scholar

- Research which is freely available for redistribution 